\title{
Simultaneously increased expression of glucocorticoid-induced tumor necrosis factor receptor and its ligand contributes to increased interleukin-5/13-producing group 2 innate lymphocytes in murine asthma
}

\author{
MENGYING ZHANG $^{1 *}$, JIE WAN $^{1 *}$, YUNYUN XU $^{1}$, DANYI ZHANG ${ }^{1,2}$, JINGJING PENG $^{1}$, \\ CHEN QI $^{1}$, QI GUO $^{1}$, SHENG XIA ${ }^{1}$, ZHAOLIANG SU ${ }^{1}$, SHENGJUN WANG ${ }^{1,2}$ and HUAXI XU ${ }^{1}$ \\ ${ }^{1}$ Department of Immunology, School of Medicine, Jiangsu University, Zhenjiang, Jiangsu 212013; ${ }^{2}$ Department of \\ Laboratory Medicine, The Affiliated People's Hospital of Jiangsu University, Zhenjiang, Jiangsu 212001, P.R. China
}

Received April 6, 2016; Accepted February 9, 2017

DOI: $10.3892 / \mathrm{mmr} .2017 .6500$

\begin{abstract}
Glucocorticoid-induced tumor necrosis factor receptor (GITR) is expressed at high levels on CD4+CD25+ regulatory $\mathrm{T}$ cells (Tregs). Following activation by its ligand (GITRL), GITR influences the activity of effector T cells and Tregs and participates in the development of numerous autoimmune and inflammatory diseases, including asthma. However, the GITR/GITRL expression level in lung tissue and its influence on group 2 innate lymphocytes (ILC2s) in asthma remains unclear. The present study detected the number of ILC2s and the expression levels of GITR and GITRL in the lung tissues of asthmatic mice by flow cytometry analysis, immunofluorescence staining and reverse transcription quantitative polymerase chain reaction. The results demonstrated that the number of ILC2s and the expression levels of ILC2-associated molecules (interleukin-33 receptor ST2, RAR related orphan receptor $A$ and inducible $T$ cell costimulator) were increased in the lung tissues of asthmatic mice. The upregulated ILC2s were accompanied by an increased number of GITR-positive cells in the spleen and lung tissues, and additionally an increased level of GITRL mRNA in lung tissue in asthma. In addition, increased mRNA expression levels of interleukin (IL)-5 and IL-13 were observed in the asthmatic lung, and there was a
\end{abstract}

Correspondence to: Professor Huaxi $\mathrm{Xu}$ or Dr Zhaoliang Su, Department of Immunology, School of Medicine, Jiangsu University, 301 Xuefu Road, Zhenjiang, Jiangsu 212013, P.R. China

E-mail: xuhx@ujs.edu.cn

E-mail:szl30@yeah.net

*Contributed equally

Key words: group 2 innate lymphocytes, glucocorticoid-induced tumor necrosis factor receptor, glucocorticoid-induced tumor necrosis factor receptor ligand, asthma, mice significant, positive correlation between the mRNA levels of GITR/GITRL and ILC2-associated molecules. Therefore, GITRL treatment may increase the number of ILC2s and/or GITR-positive cells in lung tissue. These results indicated that the activity of GITR-expressing ILC2s may be enhanced via interaction of GITRL and GITR, which may contribute to pathogenesis of asthma. These findings present potential therapeutic targets for the treatment of asthma.

\section{Introduction}

Allergic asthma is a type of allergic disease that is characterized by acute, reversible obstruction of the airway, airway hyperresponsiveness (AHR) to bronchospasmogenic compounds, airway inflammation and airway remodeling. It is established that Thelper 2 cells (Th2) are critical for the induction of allergic asthma manifestations by producing cytokines and inducing B-cell class-switching to the isotope immunoglobulin $(\mathrm{Ig}) \mathrm{E}$. Neill et al $(1,2)$ identified a novel interleukin (IL)-13-producing innate cell, termed nuocytes or group 2 innate lymphocytes (ILC2s), which are located in fat-associated lymphoid clusters. These cells were defined as lineage (Lin; lymphocyte, macrophage, dendritic cell, basophil, eosinophil, mast cell and natural killer cell)-negative and inducible $\mathrm{T}$ cell costimulatory (ICOS) -positive and additionally express the IL-25 receptor (IL-17RB) and the IL-33 receptor [T1/ST2)] (3). ILC2s are induced to secrete IL-5 and IL-13; however, limited IL-4 secretion is induced by IL-25 and IL-33 (2). In addition, ILC2s provide an essential source of IL-13 for $N$. brasiliensis worm expulsion (1). There is a limited understanding of the role of ILC2s in allergic asthma currently; however, IL-13 is essential for various aspects of the allergic lung response, including AHR. Therefore, investigation into ILC2s as a non-T-cell source of IL-13 is required. Notably, the transcription factor RAR related orphan receptor $\alpha(\operatorname{ROR} \alpha)$ is critical for the development and functioning of ILC2s (4). ILC2s have an important role in the process of Th2 response, and the polarization of ILC2s may contribute to Th2-biased differentiation. 
Regulatory T cells (Tregs) oppose effector T cell activity, and suppress AHR and allergic inflammation following stimulation with allergens in murine allergic asthma models. For example, adoptive transfer of Tregs into allergen-sensitized mice downregulates asthma manifestations (5) and, in contrast, depletion of these cells exacerbates experimental asthma $(6,7)$. The triggering of glucocorticoid-induced tumor necrosis factor receptor (GITR) by its ligand (GITRL) has been demonstrated to prevent the suppressive function of Tregs, and to increase T cell proliferation and cytokine production (8). GITR is a type I transmembrane protein and a member of the tumor necrosis factor receptor superfamily (9-11) that has constitutively high expression on the cell surface of natural Tregs (nTregs) $(9,10)$. However, low expression levels of GITR are observed on resting naïve cluster of differentiation (CD)4+ T cells, which are upregulated following activation (10-15). However, GITR expression is additionally upregulated following the activation of CD25- effector T cells $(12,13)$. The ligand of GITR, GITRL, is expressed on macrophages, dendritic cells and B cells; however, it is not expressed on T cells (14-18). GITR stimulation was previously reported to abolish the suppressive properties of nTreg cells in vitro and in vivo $(12,17-21)$. Thus, GITR is an important molecule due to its modulatory role on regulatory and effector $\mathrm{T}$ cell functions. It has been reported that GITR signaling potentiates murine AHR via enhancing Th2 activity (11). As ILC2s contribute to hyperreactivity in asthmatic mice, it was hypothesized that GITR-GITRL may additionally influence the polarization of ILC2s. To understand the relevance of GITR-GITRL signaling and ILC2s in a murine asthma model, the present study detected the number of ILC2s and GITR or GITRL-expressing cells, analyzed the levels of cytokines associated with ILC2s in the lung tissue and investigated the effect of GITR/GITRL expression levels on ILC2s in asthmatic mice.

\section{Materials and methods}

Animals. A total of 96 specific pathogen-free male BALB/c mice (age, 6-8 weeks; weight, 18 $\pm 2 \mathrm{~g}$ ) were purchased from the Laboratory Animal Center of Yangzhou University (Yangzhou, China) and housed in plastic cages in a laminar flow cabinet with sterilized wood-chip bedding. The room temperature was maintained at $23 \pm 2^{\circ} \mathrm{C}$ and a relative humidity of $55 \pm 10 \%$ with a 12 -h light-dark cycle. All mice were provided with free access to food and water. All animal experiments were approved by the Animal Care and Ethics Committee of Jiangsu University (Jiangsu, China).

Asthma model induction and lung tissue specimen treatment. As described by Wang et al (22), 50 mice were sensitized intraperitoneally on days 1 and 11 with $50 \mu \mathrm{g}$ ovalbumin (OVA; Sigma-Aldrich; Merck KGaA, Darmstadt, Germany) and $2 \mathrm{mg}$ aluminum hydroxide gel in $0.1 \mathrm{ml}$ PBS. Sensitized mice were subsequently exposed to OVA (10 $\mathrm{mg} / \mathrm{ml}$ in PBS) inhalation challenges for 30 min every day between days 22 and 26. Control animals received PBS. The mice were assessed for allergic inflammation of the lungs $24 \mathrm{~h}$ after the final lung challenge, the peripheral blood and lung tissue specimens were collected (Fig. 1A). Asthmatic mice were confirmed by the presence of lung inflammation with HE staining (Fig. 1B) and increased IgE in serum, measured by ELISA (Mouse IgE ELISA kit; AKRIE-010; Shibayagi, Shibukawa, Japan), according to the manufacturer's protocol (Fig. 1C). Lung tissue was initially perfused with PBS and mechanically crushed, followed by digestion at $37^{\circ} \mathrm{C}$ for $2 \mathrm{~h}$ in RPMI medium with $10 \%$ fetal bovine serum (Gibco; Thermo Fisher Scientific, Inc., Waltham, MA, USA) and collagenase I $(0.25 \%)$, to acquire a single cell suspension. The cell suspension was filtrated through $40 \mathrm{~mm}$ filters for flow cytometry analysis.

Flow cytometry analysis. Single-cell suspensions $\left(2 \times 10^{6}\right.$ cells $)$ from lung tissues were incubated with fluorescein isothiocyanate-labeled Lin (22-7770-72; 1:100; eBioscience, Inc., San Diego, CA, USA), PerCP/Cy5.5-labeled ICOS (107705; 1:100; BioLegend, Inc., San Diego, CA, USA) and PE-labeled T1/ST2 (145303; 1:100; BioLegend, Inc.) antibodies at $4^{\circ} \mathrm{Cfor}$ $30 \mathrm{~min}$, and fixed with $4 \%$ paraformaldehyde for flow cytometry analysis. The FITC-IgG1 (401913; 1:200, BioLegend, Inc.), PerCP/Cy5.5-IgG1 (402027, 1:200, BioLegend, Inc.) and PE-IgG1 (12-4301, 1:200; eBioscience, Inc.) served as the isotype controls. Stained cells were analyzed with an Accuri ${ }^{\mathrm{TM}}$ C6 flow cytometer (BD Biosciences, Franklin Lakes, NJ, USA) using FlowJo software 7.6 (TreeStar, Inc., Ashland, OR, USA). ILC2s were identified as Lin-ICOS+ST2+. GITR+ cells were characterized with anallophycocyanin-labeled GITR antibody (109101; 1:200; eBioscience, Inc.) and GITRL+ cells were stained with a phycoerythrin (PE)-GITRL antibody (120305; 1:200; BioLegend, Inc.). Mouse spleen cells for flow cytometry analysis were obtained as previously described (22).

Hematoxylin and eosin $(H \& E)$ and immunofluorescence staining. Freshly obtained lung tissue of asthmatic mice was fixed in $4 \%$ paraformaldehyde for $24 \mathrm{~h}$ and embedded in paraffin. Paraffin-embedded lungs were sectioned (4-6 $\mu \mathrm{m}$ ) and stained with H\&E for morphometric analysis. GITRs in the lungs were identified by immunofluorescence staining. As described previously (23), sections for immunofluorescence staining were heated for $3 \mathrm{~h}$ in a $37^{\circ} \mathrm{C}$ incubator and dewaxed followed by antigen unmasking. Sections were subsequently blocked with $1 \%$ (weight/volume) bovine serum albumin (Sigma-Aldrich; Merck KGaA) at $37^{\circ} \mathrm{C}$ for $30 \mathrm{~min}$, stained with a primary antibody against mouse GITR (14-5874-80; 1:200; eBioscience, Inc.) and visualized with a goat-anti-rat PE-conjugated secondary antibody (sc-3738; 1:200, Santa Cruz Biotechnology, Inc., Dallas, Texas, USA). Finally, slides were stained in Hoechst 33342 for 10 min. All sections were coverslipped with neutral resin medium, visualized under an Olympus fluorescence microscope and analyzed using ImageJ software v2.1.4.7 (https://imagej.nih.gov/ij/).

RNA extraction and reverse transcription-quantitative polymerase chain reaction ( $R T-q P C R)$. Total RNA was isolated from frozen tissues or fresh lung cells using TRIzol ${ }^{\circledR}$ (Invitrogen; Thermo Fisher Scientific, Inc.) and $500 \mathrm{ng}$ total RNA was reverse transcribed using a PrimeScript RT reagent kit (Takara Bio, Inc., Otsu, Japan) according to the manufacturer's protocol. Based on GenBank sequences, the primers used in this study were designed by Primer Premier 5.0 software (Premier Biosoft International, Palo Alto, CA, USA) and synthesized by Invitrogen (Thermo Fisher Scientific, Inc.). 
Table I. Sequence and length of polymerase chain reaction primers.

\begin{tabular}{llll}
\hline Gene & \multicolumn{1}{c}{ Forward primer (5'-3') } & \multicolumn{1}{c}{ Reverse primer (5'-3') } & Length, bp \\
\hline GITR & CAAGCCAGACGCTACAAGAC & CCGCTCTCATACACCCACTT & 125 \\
GITRL & CTACGGCCAAGTGATTCCTGT & GATGATCCCCCAGTATGTGT & 220 \\
ICOS & ACTTGCAGGTGTGACCTCAT & GGCCAGTGCATAGCTAGAAT & 336 \\
T1/ST2 & TGGACAGCACCTGTTCAGT & CAGGACATCAGCCAAGAAGT & 327 \\
ROR $\alpha$ & CTGACGAGGACAGGAGTAGG & AGCCGAGGTATCTCAGTCAC & 259 \\
$\beta$-actin & TGGAATCCTGTGGCATTCATGAAAC & TAAAACGCAGCTCAGTAACAGTCCG & 349 \\
IL-5 & AGGATGCTTCTGCACTTGAG & CCTCATCGTCTCATTGCTTG & 144 \\
IL-13 & TGAGCAACATCACACAAGACC & AGGCCATGCAATATCCTCTG & 157 \\
\hline
\end{tabular}

GITR, glucocorticoid-induced tumor necrosis factor receptor; GITRL, GITR ligand; ICOS, inducible T cell costimulator; ROR $\alpha$, RAR related orphan receptor 2 ; ST2, interleukin-33 receptor.

A

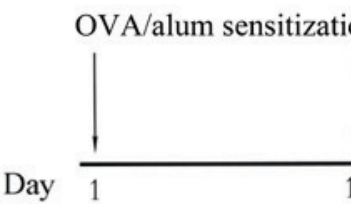

OVA inhalation challenges
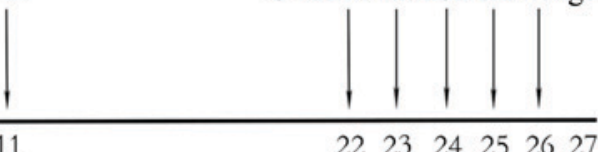

$22 \quad 2324252627$
OVA inhalation challenges

Sacrificed and measurement
B

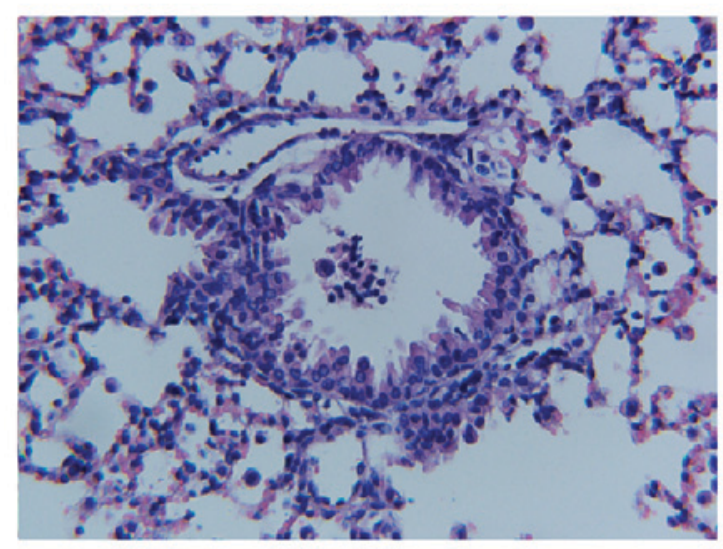

C

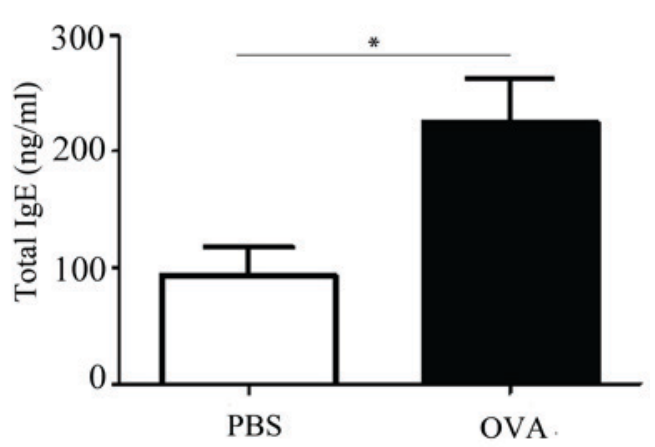

Figure 1. Establishment of a mouse model of asthma. (A) OVA stimulation process. (B) Representative images (x200) of hematoxylin and eosin-stained lung tissues from asthmatic mice, demonstrating inflammatory alterations. (C) Quantification of ELISA results indicating that increased immunoglobulin E was present in the serum of asthmatic mice. Data are presented as the mean \pm standard error. ${ }^{*} \mathrm{P}<0.05$. OVA, ovalbumin; alum, aluminum hydroxide gel.

Primer sequences are presented in Table I. Gene-specific PCR products were amplified with SYBR ${ }^{\circledR}$ Premix Ex Taq (Takara Biotechnology Co., Ltd., Dalian, China) according to the manufacturer's protocol. Briefly, $95^{\circ} \mathrm{C}$ pre-denaturation for $3 \mathrm{~min}(1 \mathrm{cycle}) ; 95^{\circ} \mathrm{C}$ denaturation for $10 \mathrm{sec}, 60^{\circ} \mathrm{C}$ annealing for $30 \mathrm{sec}, 72^{\circ} \mathrm{C}$ extension for $1 \mathrm{~min}$ (30 cycles). The levels of target gene expression were normalized to $\beta$-actin expression using the $2^{-\Delta \Delta C q}$ method (24). All samples were performed in triplicate.

Murine (m) GITRL protein and GITRL treatment. The murine GITRL fusion protein and control protein were expressed in
E. coli BL-21 with mGITRL-Pet-32a and eGFP-Pet-32a as previously described $(25,26)$, respectively. Expression was performed at $30^{\circ} \mathrm{C}$, and induced with $1 \mathrm{mmol} /$ lisopropyl $\beta$-D-1-thiogalacto-pyranoside (Sigma-Aldrich; Merck KGaA) for $6 \mathrm{~h}$, fusion proteins were purified by an immobilized affinity chromatography nickel column (Bio-Rad Laboratories, Inc., Hercules, CA, USA). The endotoxin was removed using a ToxinEraser $^{\mathrm{TM}}$ Endotoxin Removal kit (Genscript, Piscataway, NJ, USA). A total of 1-5 days prior to the first OVA challenge, the asthmatic mice were treated intravenously with $20 \mu \mathrm{g}$ purified recombinant mGITRL protein and control protein per mice per day. 
Enzyme-linked immunosorbent assay (ELISA) for plasma cytokines. Plasma IL-5 and IL-13 were measured by ELISA, according to the manufacturer's protocols (IL-5, LS-F262; eBioscience, Inc; and IL-13, EM016; Shanghai ExCell Biology, Shanghai, China). All samples were measured in triplicate using a microplate reader, and the mean concentration was calculated from the standard curve.

Statistical analysis. All statistical analyses were performed using GraphPad Prism Version 5.0 software (GraphPad Software, Inc., La Jolla, CA, USA). Data are presented as the mean \pm standard deviation. Comparisons between two groups were performed using independent-samples t-test. Pearson's correlation was used to detect the correlation between two continuous variables. $\mathrm{P}<0.05$ was considered to indicate a statistically significant difference.

\section{Results}

Enhanced GITR/GITRL expression in asthmatic mice. Flow cytometry analysis demonstrated that the percentage of GITR-positive cells in the spleen (Fig. 2A) and lung tissue (Fig. 2B) were significantly increased in asthmatic mice. Immunofluorescence staining indicated that increased GITR-positive cells were present in inflammatory lung tissue (Fig. 2C). Additionally, GITR mRNA expression in lung tissues of asthmatic mice was determined by RT-qPCR, and was demonstrated to be increased 2-fold compared with control mice (Fig. 2D). GITR and its ligand, GITRL, have been previously demonstrated to regulate the reactivity of various different cell types and to influence a variety of immunological conditions (11). Therefore, the present study detected the expression level of GITRL in the lung tissue of asthmatic mice and observed that its expression was additionally increased compared with control mice (Fig. 2E). The results indicated that GITR/GITRL signaling may be enhanced in asthmatic mice.

Increased ILC2s and ILC2-associated factors in the lung tissue of asthmatic mice. ILC2s were identified as Lin-ICOS+ST2+. The frequency of ILC2s in lung tissue from asthmatic mice was significantly increased compared with controls (Fig. 3A). $\mathrm{ROR} \alpha$ has been previously demonstrated to have a crucial function in ILC2 development and function, and ST2 is a surface marker that is expressed by and is relatively specific to ILC2s. The present study additionally detected the expression levels of ROR $\alpha$, ICOS and ST2 mRNA by using RT-qPCR. mRNA expression levels of ICOS (Fig. 3B), ST2 (Fig. 3C) and $\mathrm{ROR} \alpha$ (Fig. 3D) were significantly increased in the lung tissues of asthmatic mice compared with control mice. The results indicated that ILC2s and ILC2-associated molecules were upregulated in the lung tissue of asthmatic mice.

Enhanced expression of ILC2 function-associated cytokines in asthmatic mice. It has previously been demonstrated that IL-25 and IL-33-responsive ILC2s infiltrate the lung from peripheral blood and become a primary innate source of IL-13, and additionally produce large amounts of IL-5 (27). The present study compared gene expression levels in lung tissues from the asthmatic model and control mice by RT-qPCR,
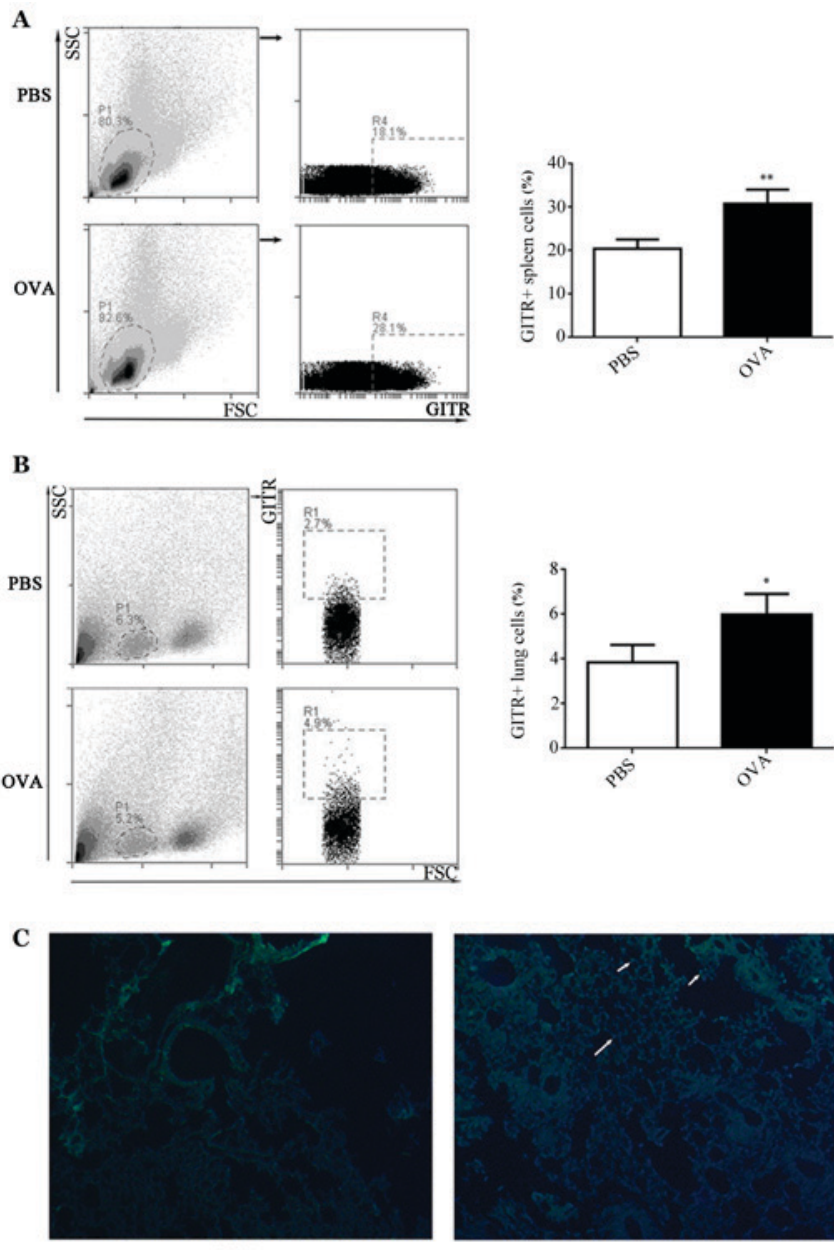

D
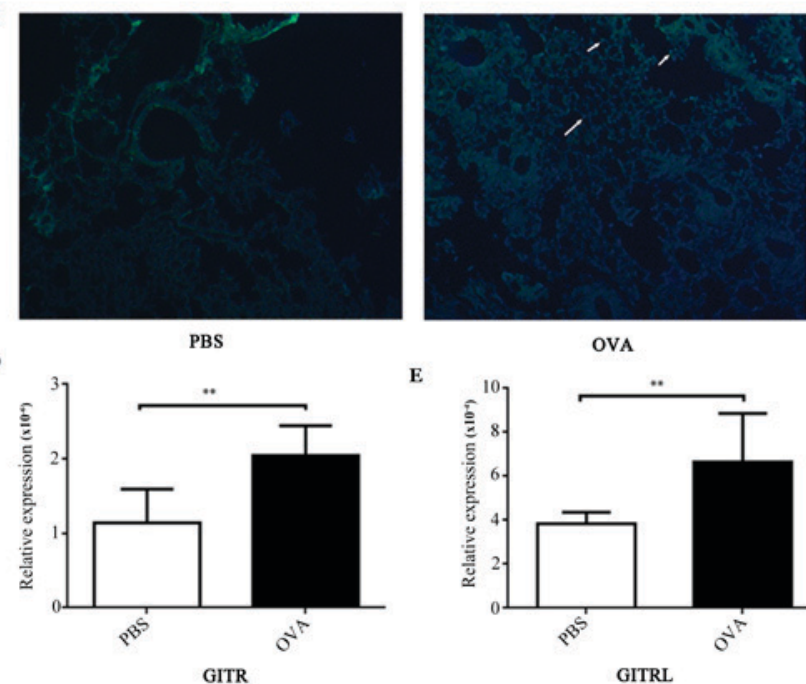

OVA

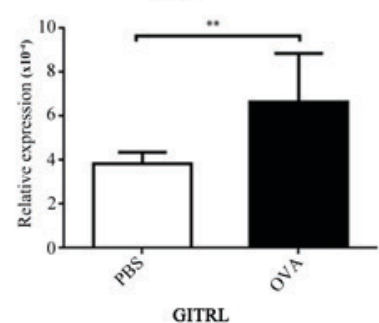

Figure 2. Levels of GITR and GITRL expression in asthmatic mice. Frequency of GITR-expressing cells was significantly increased in (A) spleen cells and (B) lung tissue from asthmatic mice compared with control mice. (C) Examination of lung slices by immunofluorescence demonstrated that increased GITR-positive cells were observed in lung tissue from asthmatic mice compared with control mice. Arrows indicate GITR-positive cells (magnification, $\mathrm{x} 40$ ). Reverse transcription-quantitative polymerase chain reaction analysis indicated that mRNA expression of (D) GITR and (E) GITRL was significantly increased in asthmatic mice compared with control mice. Data are presented as the mean \pm standard error. ${ }^{*} \mathrm{P}<0.05$ and ${ }^{* *} \mathrm{P}<0.01$. GITR, glucocorticoid-induced tumor necrosis factor receptor; GITRL, GITR ligand; OVA, ovalbumin; SSC, side scatter; FSC, forward scatter.

and demonstrated that the mRNA expression levels of IL-13 (Fig. 4A) and IL-5 (Fig. 4B) were significantly increased in asthmatic mice, which was consistent with the results of serum testing (Fig. 4C and D, respectively).

Positive correlation between mRNA levels of GITR/GITRL and ILC2-associated molecules. GITR/GITRL has an important role in regulating immune polarization conditions. To 
A
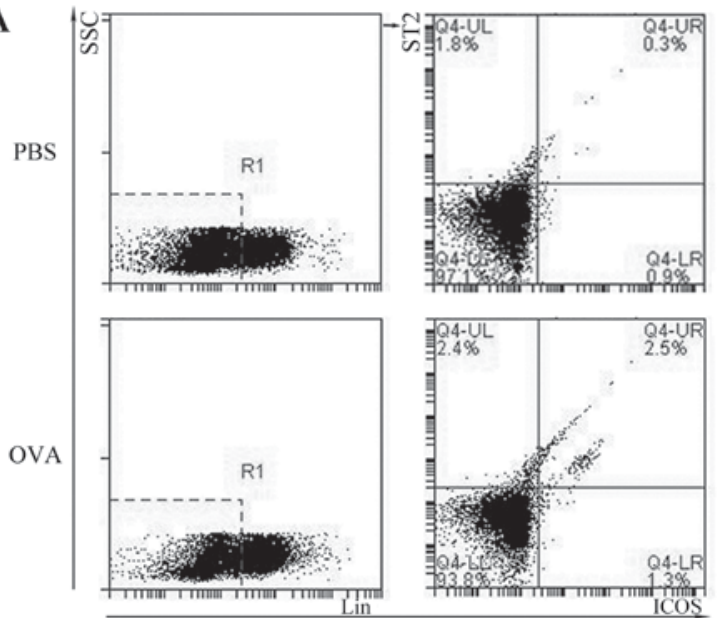
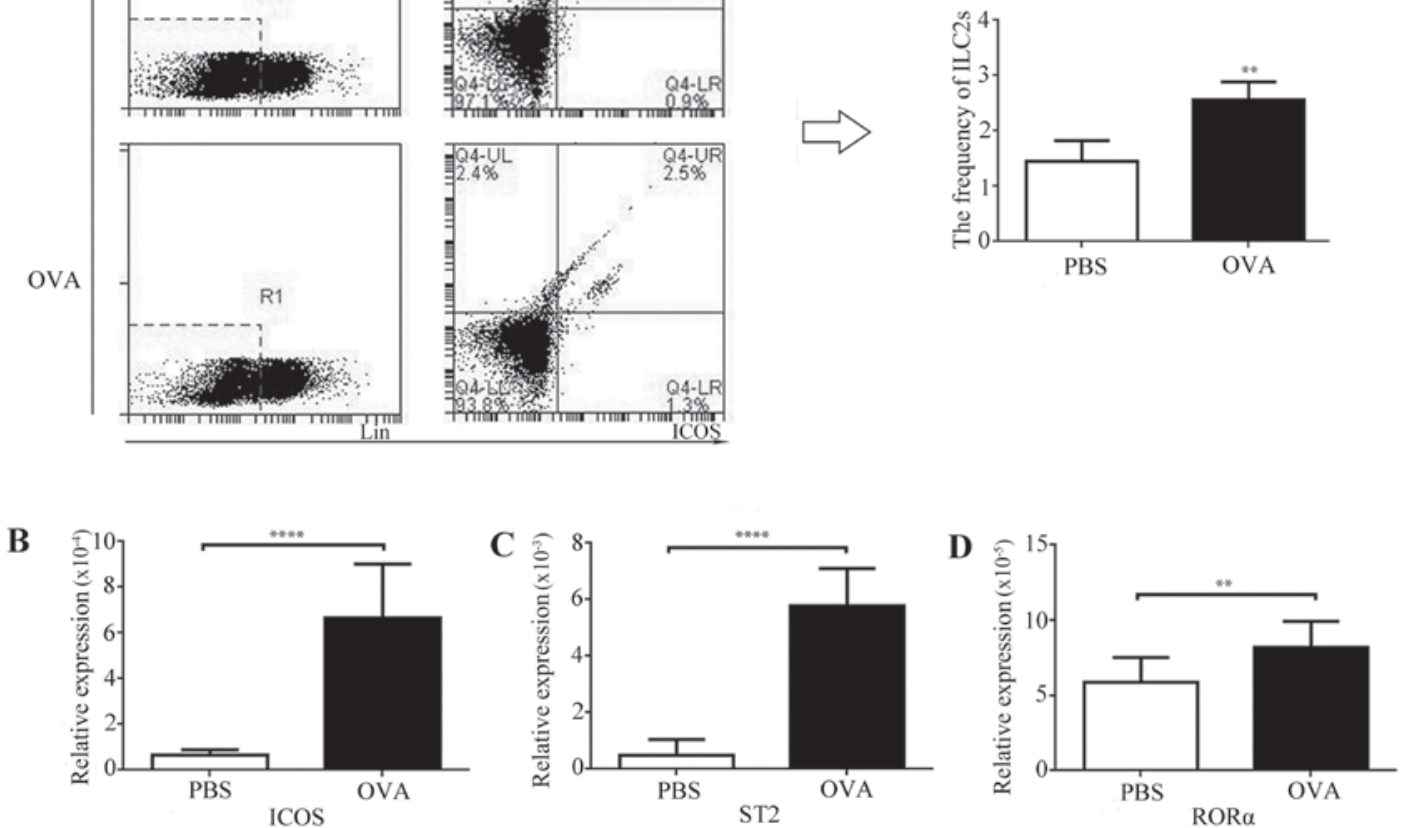

Figure 3. Increased ILC2s and ILC2-associated transcription factors in asthmatic mice. (A) The frequency of ILC2s (ST2 ${ }^{+} \mathrm{ICOS}^{+}$cells) was increased in lung tissue from asthmatic mouse model compared with control mice. Results of reverse transcription-quantitative polymerase chain reaction analysis of mRNA levels for (B) ICOS, (C) ST2 and (D) ROR $\alpha$. The data were normalized to $\beta$-actin and are presented as the mean \pm standard deviation. ${ }^{* *} \mathrm{P}<0.01$ and ${ }^{* * * * *} \mathrm{P}<0.0001$. ILC2s, group 2 innate lymphocytes; ICOS, inducible T cell costimulator; ST2, interleukin-33 receptor; ROR $\alpha$, RAR related orphan receptor 2; OVA, ovalbumin; SSC, side scatter; Lin, lineage antibody.

A

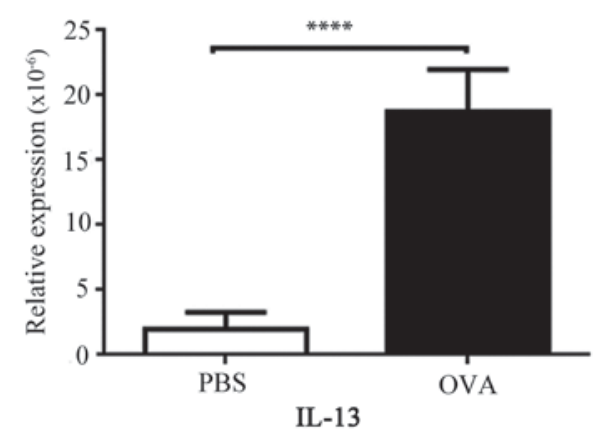

C

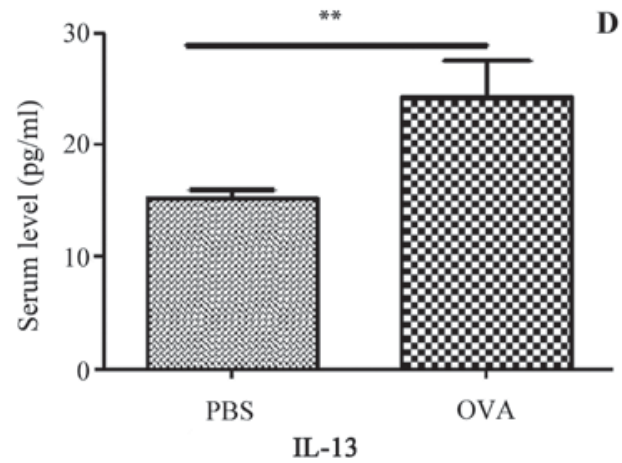

B

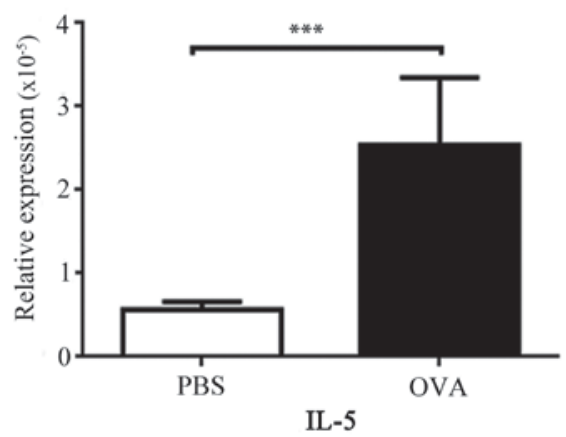

D

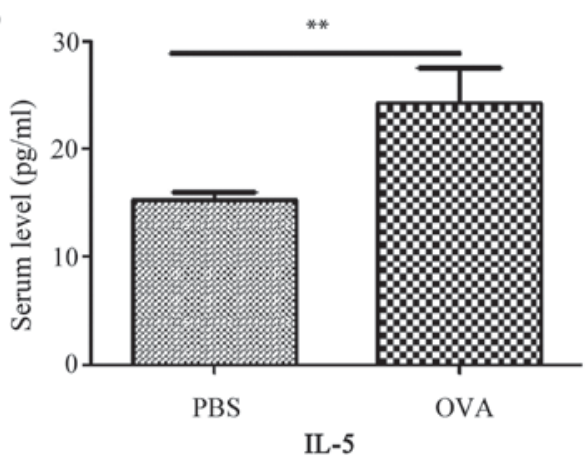

Figure 4. Expression levels of ILC2-associated cytokines IL-5 and IL-13 in lung tissues and serum of asthmatic mice. Reverse transcription-quantitative polymerase chain reaction quantification of mRNA expression levels of (A) IL-13 and (B) IL-5 demonstrated that levels were significantly increased in asthmatic mice compared with control mice. Levels of (C) IL-13 and (D) IL-5 detected in the serum. ${ }^{* *} \mathrm{P}<0.01,{ }^{* * * *} \mathrm{P}<0.001$ and ${ }^{* * * * *} \mathrm{P}<0.0001$. ILC2s, group 2 innate lymphocytes; IL, interleukin; OVA, ovalbumin. 

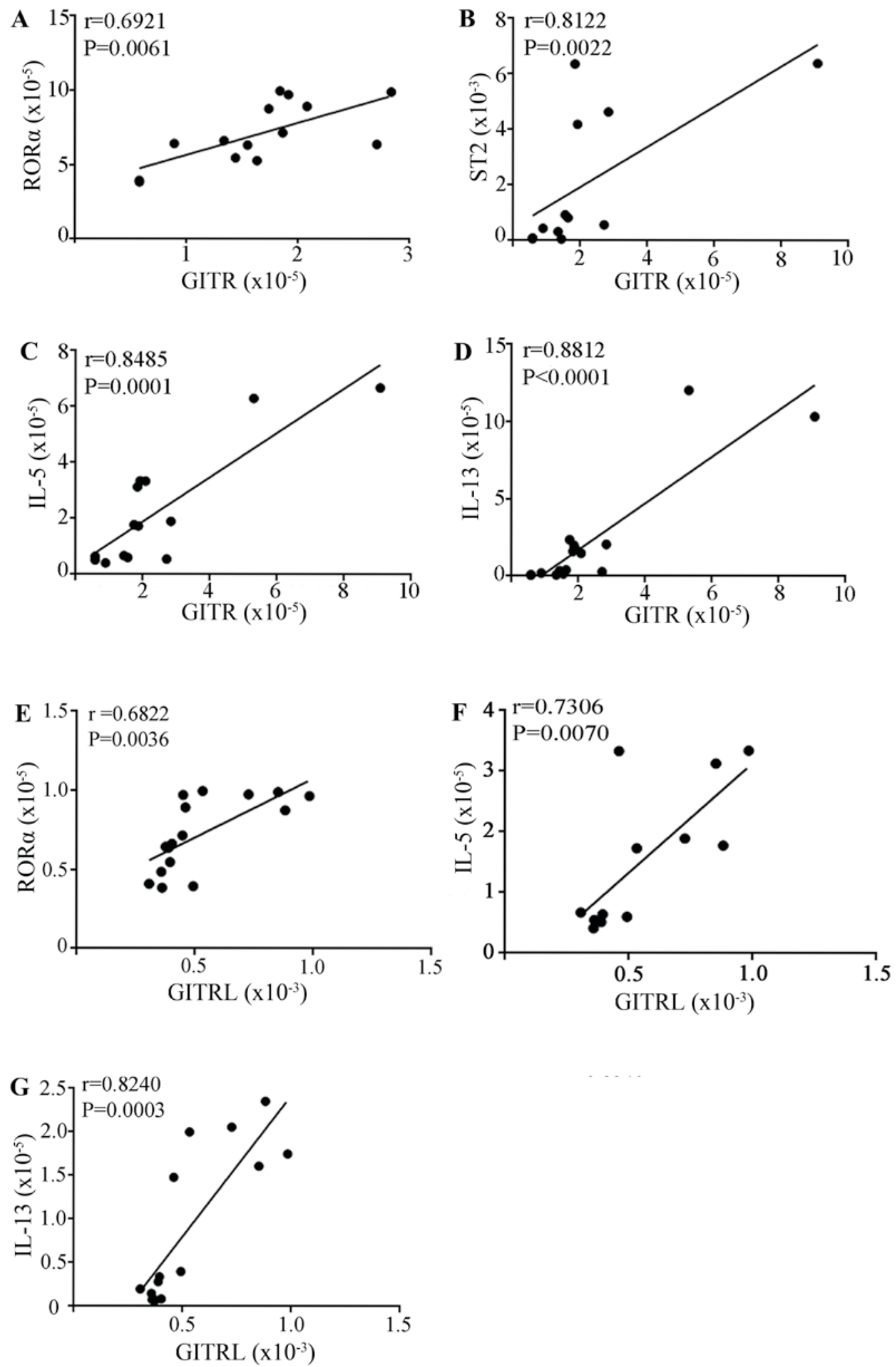

Figure 5. Correlation analysis of the expression of GITR/GITRL and ILC2-associated molecules. Correlation between the expression levels of GITR and (A) ROR $\alpha$, (B) ST2, (C) IL-5 and (D) IL-13. Correlation between the expression levels of GITRL and (E) ROR $\alpha$, (F) IL-5, (G) IL-13 expression in lung tissue from asthmatic mice. GITR, glucocorticoid-induced tumor necrosis factor receptor; GITRL, GITR ligand; ILC2s, group 2 innate lymphocytes; ROR $\alpha$, RAR related orphan receptor 2; IL, interleukin; ST2, IL-33 receptor; ICOS, inducible T cell costimulator.

understand the association between GITR and ILC2-associated molecules and cytokines in asthmatic mice, the present study analyzed the correlation between mRNA expression levels of GITR/GITRL and ROR $\alpha$, T1/ST2, IL-13, and IL-5. The results indicated that there was a significant, positive correlation between GITR and ILC2-associated molecules or signature cytokines in asthmatic mice (Fig. 5).

Expression of GITR on lung ILC2s. As presented in Fig. 3, ILC2s were located in the native lung tissue of mice and were 
A

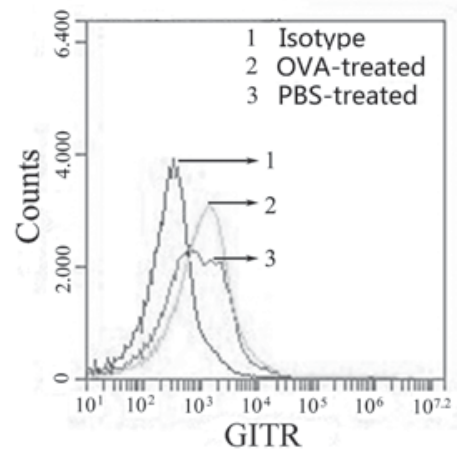

B
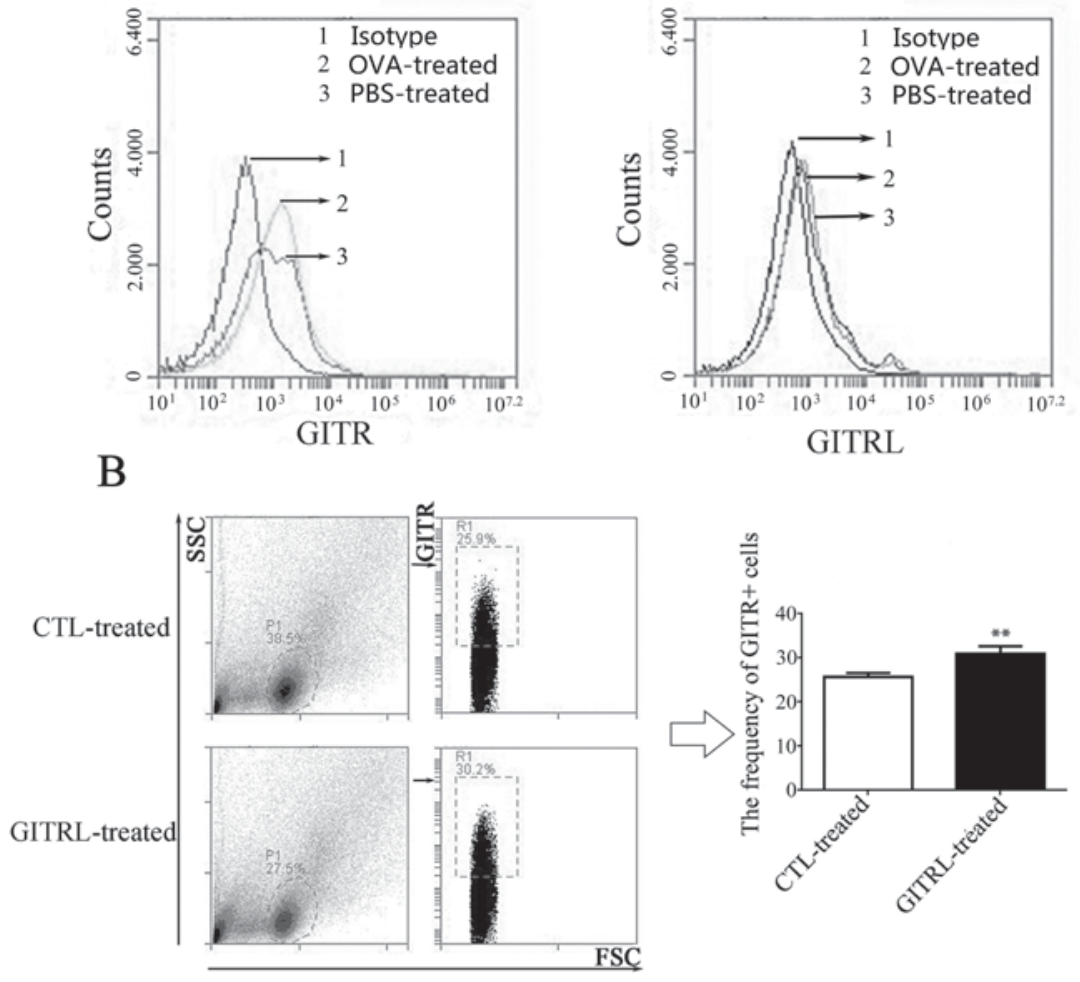

$\mathrm{C}$

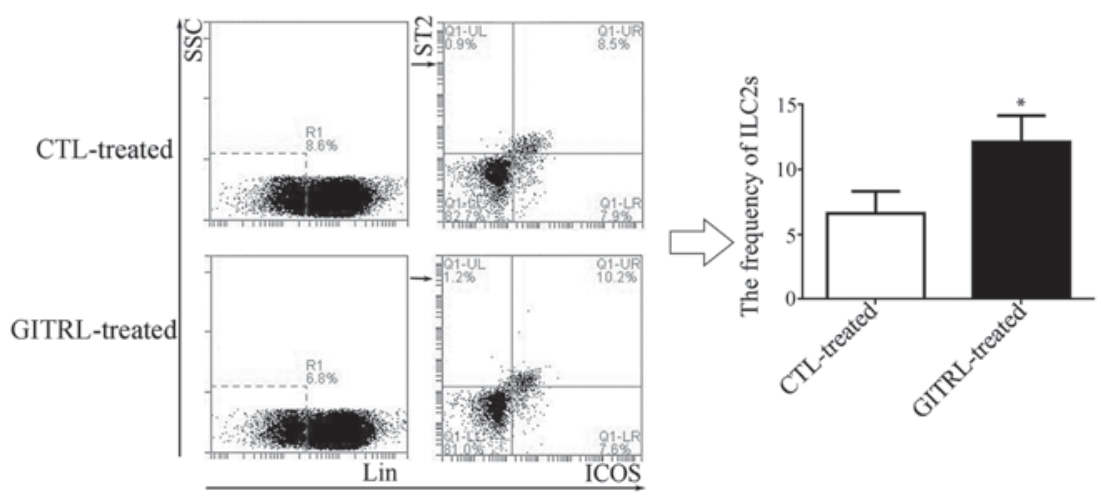

Figure 6. Frequency of GITR+ cells and ILC2s in lung tissue from GITRL-treated asthmatic mice. (A) GITR expression in lung tissue from OVA-treated mice. Left, frequency of GITR-positive cells in lung tissue from asthmatic or control mice; 1, isotype control; 2, OVA-treated mice; 3, PBS-treated mice. Increased frequency of GITR-positive cells was observed in lung tissue from asthmatic mice. Right, frequency of GITRL-positive cells in lung tissue from asthmatic or control mice; 1, isotype control; 2, OVA-treated mice; 3, PBS-treated mice. There was no difference between the 3 groups. Flow cytometry analysis indicated that the percentage of (B) GITR+ cells and (C) ILC2s (ST2 ${ }^{+} \mathrm{ICOS}^{+}$cells) was increases in lung tissue from GITRL-treated asthmatic mice compared with CTL protein-treated asthmatic mice. ${ }^{*} \mathrm{P}<0.05$ and ${ }^{* *} \mathrm{P}<0.01$. GITR, glucocorticoid-induced tumor necrosis factor receptor; ILC2s, group 2 innate lymphocytes; GITRL, GITR ligand; OVA, ovalbumin; CTL, control; SSC, side scatter; FSC, forward scatter; Lin, lineage antibody; ICOS, inducible T cell costimulator; ST2, interleukin-33 receptor.

induced by intranasal OVA administration. Once lung cells from PBS-and OVA-treated mice had been obtained, lung ILC2s were identified as Lin-ICOS+ST2+ cells and the expression of GITR and GITRL expression on resting and activated lung ILC2s was investigated by flow cytometry. As presented in Fig. 6A, different expression levels of GITR amongst control, PBS-treated and OVA-treated groups was observed; however, GITRL expression levels were more consistent in the three groups.

GITRL treatment increases the number of lung ILC2s. To determine the effect of GITRL on AHR, sensitized mice were treated intravenously with $20 \mu \mathrm{g}$ GITRL or control protein prior to the first OVA challenge, and lung ILC2s were subsequently analyzed by flow cytometry. Compared with the controls, GITRL administration induced a significant increase in the frequency of GITR+ cells (Fig. 6B) and ILC2s (Fig. 6C) in lung tissue.

\section{Discussion}

The present study, to the best of our knowledge, demonstrates for the first time that GITR- and GITRL-positive cells were 
simultaneously increased in the lung tissue of asthmatic mice, which are associated with ILC2 activation and contribute to immune imbalance, and participate in pathological mechanisms involved in asthma. Previous studies $(28,29)$ have demonstrated that GITR is primarily expressed by naive and activated T cells. In addition, CD4+ CD25+forkhead box P3+ Treg cells have been demonstrated to express high levels of GITR. GITR exerts a differential effect on Th cell proliferation and cytokine release by fully differentiated Th1 and Th2 cells in vitro; GITR increases Th2 and cytokine production; however, it does not have this effect on Th1. A similar effect on Th2 effector functions was observed in vivo in a mouse model of asthma; increased AHR, serum IgE response and cytokine production by Th2 cells was observed (11). Activation of CD4+CD25+ Tregs by the agonistic anti-GITR monoclonal antibody decreased Treg numbers (30), and reversed the suppressive activity of Treg. Previous research has demonstrated that GITRL has a wider range of tissue and cell distribution; it is not only expressed on macrophages, dendritic cells and B cells, but is additionally expressed on endothelial cells and certain stromal cells $(31,32)$. It has become clear that the functional interaction of GITRL with GITR drives T-cell activation via either co-stimulation of $\mathrm{CD} 4+$ effector $\mathrm{T}$ cells or inhibition of the suppressive function of Tregs.

It has been previously demonstrated that the inhibition of Treg function was due to the upregulation of GITR/GITRL expression (32). Notably, the present study observed that GITR was additionally expressed on ILC2s, and the expression level was significantly increased in asthmatic mice, which indicates that GITRL may have a wider range of tissue and cell distribution than previously observed. ILC2s have an important role in acute allergic lung inflammation, including asthma and allergic diarrhoea. Given their distribution and function, lung ILC2s are hypothesized to coordinate epithelial responses to the external environment; the activity of ILC2s is additionally associated with interactions with many other immune cells. Enhanced GITR expression on ILC2s was observed in the lung tissue from asthmatic mice in the present study, which led to consideration of the potential outcome of GITR-GITRL interaction. On the one hand, the function of Tregs maybe inhibited by increased GITRL in lung tissue; however, it may additionally enhance the activity of ILC2s. Allergy is a major health problem associated with the industrialized world and overproduction of Th2-type cytokines, which include certain ILs (IL-4, 5 and 9) and IgE, are involved in the type 2 immune response that is responsible for the majority of allergen-induced inflammation at mucosal surfaces (33). There is currently limited understanding of the underlying mechanisms that may be responsible for allergen-induced initiation of Th0 to Th2 differentiation during the sensitization phase. However, it is accepted that the cytokine environment has a role in dictating the differentiation of Th0 cells into different Th cell populations. Specifically, IL-4 and the transcription factor GATA binding protein 3are considered essential for Th2 cell differentiation and the production of type- 2 cytokines. However, the initial source of IL-4 is not established as multiple cell populations, including natural killer T cells and dendritic cells amongst others, may contribute. Furthermore, Th2 cell differentiation has been induced in vitro without the presence of exogenous IL-4, which indicates that a Th2 cell differentiation signaling pathway that is independent of IL-4-may exist. Halim et al (34) indicated that ILC2s induce Th2 cell differentiation, which was deduced based on increased type 2 cytokine production, increased $\mathrm{IgE}$ titers and the presence of eosinophilic lung inflammation, which was partially confirmed by our previous studies (35). The earliest studies on asthma pathology demonstrated that CD4+ T lymphocytes were present in asthma biopsies. To identify the ILC2 activity in lung tissue from asthmatic mice, the present study detected the mRNA expression levels of ILC2-associated transcription factors and cytokines. As expected, ROR $\alpha$, ICSO, IL-5 and IL-13 were significantly increased in asthmatic mice compared with control mice, which was associated with upregulated expression of GITR/GITRL, and a positive correlation was observed between GITR/GITRL expression and the expression of ILC2-associated factors. The administration of recombinant mGITRL protein induced an increase in the numbers of lung ILC2s. The results indicated that GITR-GITRL signaling may contribute to ILC2 polarization and its cytokine production in a direct or indirect way. The mechanism behind this requires further investigation.

In conclusion, to the best of our knowledge, the results of the present study demonstrated for the first time that in asthmatic mice, the number of ILC2s and GITR+/GITRL+ cells were simultaneously increased, and increased GITR expression on ILC2s was observed. There was a significant, positive correlation between GITR-GITRL expression levels and the expression of ILC2-associated molecules. Furthermore, GITRL treatment increased the number of ILC2s and/or GITR+ cells in lung tissue. These results indicated that the activity of ILC2s maybe enhanced by the interaction of GITRL and GITR, which may subsequently contribute to the pathogenesis of asthma. These findings present potential therapeutic targets for the treatment of asthma.

\section{Acknowledgements}

This study was supported by grants from the National Natural Science Foundation of China (grant nos. 31270947, 31170849 and 81370084) and the Postdoctoral Foundation of China (grant nos. 2014T70490 and 2013T60508).

\section{References}

1. Neill DR and McKenzie AN: Nuocytes and beyond: New insights into helminth expulsion. Trends Parasitol 27: 214-221, 2011.

2. Neill DR, Wong SH, Bellosi A, Flynn RJ, Daly M, Langford TK, Bucks C, Kane CM, Fallon PG, Pannell R, et al: Nuocytes represent a new innate effector leukocyte that mediates type-2 immunity. Nature 464: 1367-1370, 2010.

3. Maazi H, Patel N, Sankaranarayanan I, Suzuki Y, Rigas D, Soroosh P, Freeman GJ, Sharpe AH and Akbari O: ICOS:ICOS-ligand interaction is required for type 2 innate lymphoid cell function, homeostasis, and induction of airway hyperreactivity. Immunity 42: 538-551, 2015.

4. Wong SH, Walker JA, Jolin HE, Drynan LF, Hams E, Camelo A, Barlow JL, Neill DR, Panova V, Koch U, et al: Transcription factor ROR $\alpha$ is critical for nuocyte development. Nat Immunol 13: 229-236, 2012.

5. Kearley J, Barker JE, Robinson DS and Lloyd CM: Resolution of airway inflammation and hyperreactivity after in vivo transfer of CD4+CD25+ regulatory T cells is interleukin 10 dependent. J Exp Med 202: 1539-1547, 2005. 
6. Jaffar Z, Sivakuru T and Roberts K: CD4+CD25+ T cells regulate airway eosinophilic inflammation by modulating the Th2 cell phenotype. J Immunol 172: 3842-3849, 2004.

7. Lewkowich IP, Herman NS, Schleifer KW, Dance MP, Chen BL, Dienger KM, Sproles AA, Shah JS, Köhl J, Belkaid Y and Wills-Karp M: CD4+CD25+ T cells protect against experimentally induced asthma and alter pulmonary dendritic cell phenotype and function. J Exp Med 202: 1549-1561, 2005.

8. Placke T, Kopp HG and Salih HR: Glucocorticoid-induced TNFR-related (GITR) protein and its ligand in antitumor immunity: Functional role and therapeutic modulation. Clin Dev Immunol 2010: 239083, 2010.

9. Joetham A, Ohnishi H, Okamoto M, Takeda K, Schedel M, Domenico J, Dakhama A and Gelfand EW: Loss of T regulatory cell suppression following signaling through glucocorticoid-induced tumor necrosis receptor (GITR) is dependent on c-Jun N-terminal kinase activation. J Biol Chem 287: 17100-17108, 2012.

10. Lommatzsch M, Julius P, Kuepper M, Garn H, Bratke K, Irmscher S, Luttmann W, Renz H, Braun A and Virchow JC: The course of allergen-induced leukocyte infiltration in human and experimental asthma. J Allergy Clin Immunol 118: 91-97, 2006.

11. Motta AC, Vissers JL, Gras R, VanEsch BC, Van Oosterhout AJ and Nawijn MC: GITR signaling potentiates airway hyperresponsiveness by enhancing Th2 cell activity in a mouse model of asthma. Respir Res 10: 93, 2009.

12. Shimizu J, Yamazaki S, Takahashi T, Ishida Y and Sakaguchi S: Stimulation of CD25(+)CD4(+) regulatory T cells through GITR breaks immunological self-tolerance. Nat Immunol 3: 135-142, 2002.

13. McHugh RS, Whitters MJ, Piccirillo CA, Young DA, Shevach EM, Collins M and Byrne MC: CD4(+)CD25(+) immunoregulatory T cells: Gene expression analysis reveals a functional role for the glucocorticoid-induced TNF receptor. Immunity 16: 311-323, 2002.

14. Tone M, Tone Y, Adams E, Yates SF, Frewin MR, Cobbold SP and Waldmann H: Mouse glucocorticoid-induced tumor necrosis factor receptor ligand is costimulatory for T cells. Proc Nat Acad Sci USA 100: 15059-15064, 2003

15. Yu KY, Kim HS, Song SY, Min SS, Jeong JJ and Youn BS: Identification of a ligand for glucocorticoid-induced tumor necrosis factor receptor constitutively expressed in dendritic cells. Biochem Biophys Res Commun 310: 433-438, 2003.

16. Kim JD, Choi BK, Bae JS, Lee UH, Han IS, Lee HW, Youn BS, Vinay DS and Kwon BS: Cloning and characterization of GITR ligand. Genes Immun 4: 564-569, 2003.

17. Ronchetti S, Ricci E, Petrillo MG, Cari L, Migliorati G, Nocentini G and Riccardi C: Glucocorticoid-induced tumour necrosis factor receptor-related protein: A key marker of functional regulatory T cells. J Immunol Res 2015: 171520, 2015.

18. KanamaruF, YoungnakP,Hashiguchi M,Nishioka T, TakahashiT, Sakaguchi S, Ishikawa I and Azuma M: Costimulation via glucocorticoid-induced TNF receptor in both conventional and CD25+ regulatory CD4+ T cells. J Immunol 172: 7306-7314, 2004.

19. Kohm AP, Williams JS and Miller SD: Cutting edge: Ligation of the glucocorticoid-induced TNF receptor enhances autoreactive CD4+ T cell activation and experimental autoimmune encephalomyelitis. J Immunol 172: 4686-4690, 2004.

20. Stephens GL, McHugh RS, Whitters MJ, Young DA, Luxenberg D, Carreno BM, Collins M and Shevach EM: Engagement of glucocorticoid-induced TNFR family-related receptor on effector $\mathrm{T}$ cells by its ligand mediates resistance to suppression by CD4+CD25+ T cells. J Immunol 173: 5008-5020, 2004
21. Zhan Y,Funda DP,Every AL, Fundova P, Purton JF, Liddicoat DR, Cole TJ, Godfrey DI, Brady JL, Mannering SI, et al: TCR-mediated activation promotes GITR upregulation in T cells and resistance to glucocorticoid-induced death. Int Immunol 16: 1315-1321, 2004.

22. Wang SY, Yang M, Xu XP, Qiu GF, Ma J, Wang SJ, Huang XX and Xu HX: Intranasal delivery of T-bet modulates the profile of helper $\mathrm{T}$ cell immune responses in experimental asthma. J Investig Allergol Clin Immunol 18: 357-365, 2008.

23. He Z, Shotorbani SS, Jiao Z, Su Z, Tong J, Liu Y, Shen P, Ma J, Gao J, Wang T, et al: HMGB1 promotes the differentiation of Th17 via up-regulating TLR2 and IL-23 of CD14+ monocytes from patients with rheumatoid arthritis. Scand J Immunol 76: 483-490, 2012

24. Livak KJ and Schmittgen TD: Analysis of relative gene expression data using real-time quantitative PCR and the 2(-Delta Delta C(T)) Method. Methods 25: 402-408, 2001.

25. Zhengjun H, Shengjun W, Yanping Z, Junfeng B, Jia T, Jie M, Jun C, Changgui S and Huaxi X: Construction of eukaryotic expression plasmid containing mGITRL gene and its expression in Lewis cell. J Jiangsu Univ (Medicine Edition) 18: 107-110, 2008.

26. Shengjun W, Bin M, Jia T, Huaxi H and Shengli Y: Cloning and sequence analysis of mouse glucocorticoid-induced tumor necrosis factor receptor ligand gene. J Jiangsu Univ (Medicine Edition) 14: 97-99, 2004.

27. Martinez-Gonzalez I, Steer CA and Takei F: Lung ILC2s link innate and adaptive responses in allergic inflammation. Trends Immunol 36: 189-195, 2015.

28. Wenzel SE: Asthma: Defining of the persistent adult phenotypes. Lancet 368: 804-813, 2006.

29. Ronchetti S, Zollo O, Bruscoli S, Agostini M, Bianchini R, Nocentini G, Ayroldi E and Riccardi C: GITR, a member of the TNF receptor superfamily, is costimulatory to mouse T lymphocyte subpopulations. Eur J Immunol 34: 613-622, 2004.

30. Wang S, Shi Y, Yang M, Ma J, Tian J, Chen J, Mao C, Jiao Z, Ko KH, Baidoo SE, et al: Glucocorticoid-induced tumor necrosis factor receptor family-related protein exacerbates collagen-induced arthritis by enhancing the expansion of Th17 cells. Am J Pathol 180: 1059-1067, 2012.

31. Olsen PC, Kitoko JZ, Ferreira TP, de-Azevedo CT, Arantes AC and Martins MA: Glucocorticoids decrease Treg cell numbers in lungs of allergic mice. Eur J Pharmacol 747: 52-58, 2015.

32. Nocentini $G$ and Riccardi C: GITR: A modulator of immuneresponse and inflammation. Adv Exp Med Biol 647: 156-173, 2009.

33. Oczypok EA, Milutinovic PS, Alcorn JF, Khare A, Crum LT, Manni ML, Epperly MW, Pawluk AM, Ray A and Oury TD: Pulmonary receptor for advanced glycation end-products promotes asthma pathogenesis through IL-33 and accumulation of group 2 innate lymphoid cells. J Allergy Clin Immunol 136: 747-756, 2015.

34. Halim TY, Steer CA, Mathä L, Gold MJ, Martinez-Gonzalez I, McNagny KM, McKenzie AN and Takei F: Group 2 innate lymphoid cells are critical for the initiation of adaptive T helper 2 cell-mediated allergic lung inflammation. Immunity 40: 425-435, 2014.

35. Wu Y, Yan Y, Su Z, Bie Q, Wu J, Wang S, Yu Y, Ding H, Lu P and $\mathrm{Xu} \mathrm{H}$ : Enhanced circulating ILC2s accompany by upregulated MDSCs in patients with asthma. Int J Clin Exp Pathol 8: 3568-3579, 2015 . 